\title{
COMET experiment:
}

\section{- A search for muon-to-electron conversion at} J-PARC -

\author{
Hajime Nishiguchi ${ }^{* \dagger}$ \\ High Energy Accelerator Research Organization, KEK \\ E-mail: hajime.nishiguchi@kek.jp
}

The COMET Experiment at J-PARC aims to search for the lepton-flavour violating (LFV) process of muon to electron conversion in a muonic atom, $\mu^{-} N \rightarrow \mathrm{e}^{-} N$, with a branching-ratio sensitivity of $6 \times 10^{17}$, which is 4 orders of magnitude better than the present upper limit. Complemental searches for two kinds of muon LFV decay modes, $\mu^{+} \rightarrow \mathrm{e}^{+} \gamma$ and $\mu^{-} N \rightarrow \mathrm{e}^{-} N$, are quite important in order to explore the parameter region predicted by most well-motivated theoretical models beyond the Standard Model such as SUSY-GUT, seesaw, little-Higgs, etc. MEG experiment reports the latest result on $\mu^{+} \rightarrow \mathrm{e}^{+} \gamma$ search at PSI and makes a strong limit on new physics models, i.e. filling in the missing peace of muon $\mathrm{LFV}, \mu^{-} N \rightarrow \mathrm{e}^{-} N$, will certainly play an important role. The need for this sensitivity places several stringent requirements on both the muon beam and the detector system. In order to realise the experiment effectively, a staged approach to deployment is endorsed by the J-PARC Program Advisory Committee and KEK, and the "COMET Phase-I" experiment will commence its engineering runs in 2018. The construction of experimental facility, beam line, magnets and detectors has been already started. The current $\mathrm{R} \& \mathrm{D}$ and construction status and prospects of the experiment are presented in addition to the experimental overview.

The European Physical Society Conference on High Energy Physics 22-29 July 2015

Vienna, Austria

${ }^{*}$ Speaker.

${ }^{\dagger}$ On behalf of the COMET collaboration 


\section{Introduction}

A Lepton Flavour Violation (LFV) among charged leptons, eg. $\mu^{-} N \rightarrow \mathrm{e}^{-} N$ process etc., which has never been observed while the quark mixing and the neutrino oscillations have been experimentally confirmed, is attracting a great deal of attention, since its observation is highly expected by most of well-motivated theories beyond the Standard Model[1]. It is predicted that $\mu^{-} N \rightarrow \mathrm{e}^{-} N$ is naturally causable with a branching ratio just below the current upper bound, $10^{-13} \sim 10^{-16}$, by leading theories for physics beyond the standard model, e.g. Supersymmetric theories of Grand Unification or Supersymmetric Standard Model with seesaw mechanism (eg. see Ref.[2,3] for a review). The ambitious goal of the COMET experiment (COherent Muon to Electron Transition) [4] is searching for a $\mu^{-} N \rightarrow \mathrm{e}^{-} N$ process with an improved sensitivity by at least four orders of magnitude over the last best upper limit on a $\mu^{-} N \rightarrow \mathrm{e}^{-} N$ branching ratio reported by the SINDRUM-II collaboration, $7 \times 10^{-13}$ [5].

\section{2. $\mu^{-} N \rightarrow \mathbf{e}^{-} N$ Search Experiment}

The most prominent muon lepton flavour violating process is coherent neutrino-less conversion of a negative muon to an electron in a muonic atom $\left(\mu^{-} N \rightarrow \mathrm{e}^{-} N\right)$. When a negative muon is stopped in some material, it is trapped by an atom, and a muonic atom is formed. After it cascades down energy levels in the muonic atom, the muon is bound in its $1 s$ ground state. The fate of the muon is then either decay in orbit (DIO) $\left(\mu^{+} \rightarrow \mathrm{e}^{+} \bar{v}_{\mu} v_{\mathrm{e}}\right)$ or nuclear muon capture by a nucleus $N(A, Z)$ of mass number $A$ and atomic number $Z$, namely, $\mu^{-}+N(A, Z) \rightarrow v_{\mu}+N(A, Z-1)$. However, in the context of lepton flavour violation in physics beyond the Standard Model, the exotic process of neutrino-less muon capture, such as $\mu^{-}+N(A, Z) \rightarrow \mathrm{e}^{-}+N(A, Z)$ is also expected. This process is called muon-to-electron conversion in muonic atom. This process violates the conservation of lepton flavour numbers, but the total number of lepton is conserved.

The event signature of coherent $\mu^{-} N \rightarrow \mathrm{e}^{-} N$ conversion in a muonic atom is a monochromatic single electron emitted from the conversion with an energy of $E_{\mu \mathrm{e}}=m_{\mu}-B_{\mu}-E_{\text {recoil }}$, where $m_{\mu}$ is the muon mass, and $B_{\mu}$ is the binding energy of the $1 s$ muonic atom. $E_{\text {recoil }}$ is the nuclear recoil energy which is extremely small and negligible. Since $B_{\mu}$ depends on nuclei $E_{\mu \mathrm{e}}$ varies, eg. 104.3 MeV for Ti and 105.1 MeV for Al.

From an experimental point of view, $\mu^{-} N \rightarrow \mathrm{e}^{-} N$ conversion is a very attractive process for the following reasons: (1) First, the energy of the signal electron of about $105 \mathrm{MeV}$ is far above the end-point energy of the normal muon decay spectrum $(\sim 52.8 \mathrm{MeV})$. (2) Secondly, since the event signature is a monochromatic electron, no coincidence measurement is required. The search for this process has the potential to improve sensitivity by using a high muon rate without suffering from accidental background events, which would be serious for other processes, such as $\mu^{+} \rightarrow \mathrm{e}^{+} \gamma$ decay search.

There are several potential sources of electron background events in the energy region around $100 \mathrm{MeV}$, which can be grouped into three categories as follows. The first group is intrinsic physics backgrounds which come from muons stopped in the muon-stopping target. The second is beamrelated backgrounds which are caused by beam particles of muons and other contaminated particles 


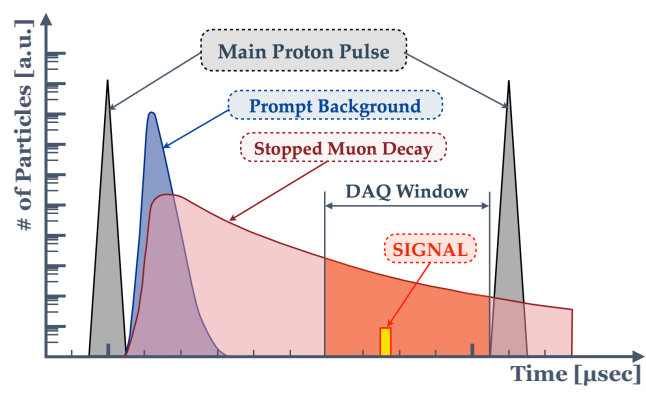

Figure 1: Time structure of the proton beam, background events and DAQ window for $\mu^{-} N \rightarrow \mathrm{e}^{-} N$ search experiment

in a muon beam. The third is other backgrounds which are, for instance, cosmic-ray backgrounds, and fake tracking events, and so on.

In order to suppress backgrounds, in particular beam-related backgrounds, the following key elements have been proposed as the next generation of $\mu^{-} N \rightarrow \mathrm{e}^{-} N$ conversion search experiments ${ }^{1}$.

(A) Beam Pulsing: Since muonic atoms have lifetimes of the order of $1 \mu \mathrm{sec}$, a pulsed beam with its width that is enough short compared with these lifetimes would allow one to remove beam-related backgrounds by performing measurements in a delayed DAQ window. To eliminate prompt beam-related backgrounds, excellent extinction of proton beam is required during the DAQ interval. The relation between the required time structure of proton beam and the DAQ window for $\mu^{-} N \rightarrow \mathrm{e}^{-} N$ search is schematically shown in Figure 1 .

(B) Pion Capture System with a High Solenoid Field: Superconducting solenoidal magnets of a high magnetic field surrounding a proton target to capture pions in a large solid angle, called pion capture system, leads to a dramatic increase of muon yields by several orders of magnitude.

(C) Curved Solenoids for Muon Transport: The curved solenoids are adopted to select charges and momenta of muons as well as removing neutral particles in a beam. In a curved solenoidal magnetic field, a centre of the helical trajectory of a charged particle is shifted perpendicular to the curved plane. The shift, whose amount is given as a function of momentum and its charge, makes a dispersive beam.

The COMET experiment[4] at the Japan Proton Accelerator Research Complex (J-PARC) was proposed in order to realize above very innovative ideas.

\section{The COMET Experiment}

The COMET uses a proton beam of $56 \mathrm{~kW}$ which will be available at J-PARC main ring (MR) accelerator. The aimed sensitivity at COMET is better than $10^{-16}$ which would explore most of the region predicted by leading theories for physics beyond the standard model. A schematic layout of the COMET experiment is presented in Figure 2. The COMET plans to use an $8 \mathrm{GeV}, 7 \mu \mathrm{A}$, slow extracted proton beam from the J-PARC MR. This equates to a beam power of $56 \mathrm{~kW}$ which

\footnotetext{
${ }^{1}$ They are based on the ideas developed in the MELC proposal at the Moscow Meson Factory [6].
} 


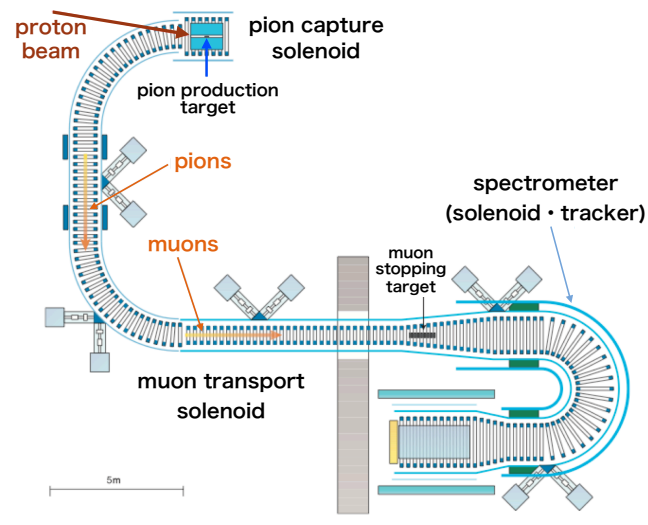

Figure 2: A schematic layout of the COMET experiment

is much less than the design power of $750 \mathrm{~kW}$. A proton beam is used to produce pions, which are then captured and transported by a series of superconducting solenoids. The pions decay into muons as they travel along the muon transport channel. The toroidal field of the muon transport channel selects muons with negative charge and momentum less than $75 \mathrm{MeV} / c$. The mon stopping target slows the muons down until they are captured in nuclear orbit by a nucleus in the target. A muon could then convert into an electron, with a momentum of around $105 \mathrm{MeV} / c$, or it could decay normally producing an electron with lower momentum. The electron spectrometer selects electrons of interest, collimating off electrons not of interest at the exit of the spectrometer. The momentum and energy of the electron is then measured by the tracker and calorimeter, respectively. The tracking detector is located in a uniform, $1 \mathrm{~T}$, magnetic field within the vacuum of the detector solenoid. It is designed to measure with good efficiency, the parameters of the helical trajectory of electrons that are emitted from the muon stopping target. The detector consists of a set of 5 straw tracker layers, $48 \mathrm{~cm}$ apart, and placed so that the axial direction of the straws is transverse to the axis of the solenoid. Each of the five tracker layers consists of four planes, two to measure the $x$ coordinate and two to measure the $y$ coordinate. Each pair of planes is staggered by half a straw diameter in order to solve left-right ambiguities. A calorimeter is positioned behind the tracker, and consists of LYSO $\left(\mathrm{Lu}_{1.8} \mathrm{Y}_{0.2} \mathrm{SiO}_{5}(\mathrm{Ce})\right.$ ) crystal viewed by APD (avalanche photo diodes). Electrons which pass through the tracker are absorbed in the calorimeter and if the deposited energy lies within a window about $105 \mathrm{MeV}$, a trigger signal is generated.

\section{Staged Approach}

The COMET experiment is planned to be carried out separately by two stages, called "PhaseI" and "Phase-II". The COMET Phase-I will construct the pion-capture system and the curved solenoid up to the first $90^{\circ}$ bending and will place the detector at the exit of this first bending ahead of the "full COMET", called phase-II, as schematically shown in Figure 3. There are two main purposes to perform COMET Phase-I ahead of the Phase-II.

- Measure the Background at the $1 \mathrm{st} 90^{\circ}$ bending 


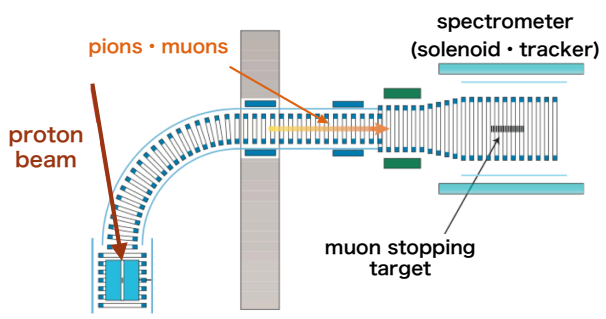

Figure 3: Phase-I Experiment of the COMET Staging Approach

- Perform the $\mu$-e search at the 1 st $90^{\circ}$ bending

Concerning the first purpose, so far the experimental optimization, not only for beam-line design but also for detector design, is completely based on the simulation. However, the hadron production code, eg. MARS[7] etc., needs to be verified with real data for such an extremely high dose environment. Phase-I background measurement will provide the significant input for such simulation framework, and give a further optimization for experiment which will guarantee the successful conclusion of experiment. Concerning the second purpose, even at the first $90^{\circ}$ bending one can perform the search experiment for $\mu^{-} N \rightarrow \mathrm{e}^{-} N$ conversion. Of course, this measurement cannot achieve the unprecedented sensitivity of final COMET experiment. However, one can achieve much better sensitivity than the current experimental upper bound, at least two orders of magnitude better than the current bound, thanks to the good pulsing and high intensity muon beam which is realized by J-PARC accelerator. For the $\mu^{-} N \rightarrow \mathrm{e}^{-} N$ search at Phase-I, Cylindrical Drift Chamber (CDC) system is used for the electron tracker in order to maximise the detector acceptance ${ }^{2}$. A comparison between Phase-I and Phase-II is summarized in Table 1.

Table 1: A comparison between COMET "Phase-I" and "Phase-II"

\begin{tabular}{lcc}
\hline \hline & Phase-I & Phase-II \\
\hline Proton Beam Power & $3 \mathrm{~kW}$ & $56 \mathrm{~kW}$ \\
Measurement Span & $9.9 \times 10^{6} \mathrm{~s}$ & $2.0 \times 10^{7} \mathrm{~s}$ \\
Number of Proton & $2.4 \times 10^{19}$ & $8.5 \times 10^{20}$ \\
Number of Stopped Muon & $1.2 \times 10^{16}$ & $2.0 \times 10^{18}$ \\
Number of BG events & 0.02 & 0.3 \\
Single Event Sensitivity (SES.) & $3.1 \times 10^{-15}$ & $2.6 \times 10^{-17}$ \\
Branching Ratio Upper Limit (90\% CL.) & $7.0 \times 10^{-15}$ & $6.0 \times 10^{-17}$ \\
Will be performed in & $2018 / 19$ & $2021 \sim$ \\
\hline \hline
\end{tabular}

\footnotetext{
${ }^{2}$ Only $90^{\circ}$ bending and short transport line is not long enough to suppress severe backgrounds in the transported beam particles so that the centre part of the transported beam cannot be used for $\mu^{-} N \rightarrow \mathrm{e}^{-} N$ search experiment. In order to avoid a reduction of acceptance due to this issue, muon stopping target would be placed at the centre of tracking volume. This is the reason why Phase-I needs the cylindrical drift chamber.
} 


\section{Current Status}

Recently the facility construction is ongoing at a rapid rate. As shown in Figure 4 (Left), the facility building was already completed by extracting the present hadron experimental facility of J-PARC. In the hall, beam line construction is underway including the proton beam line which
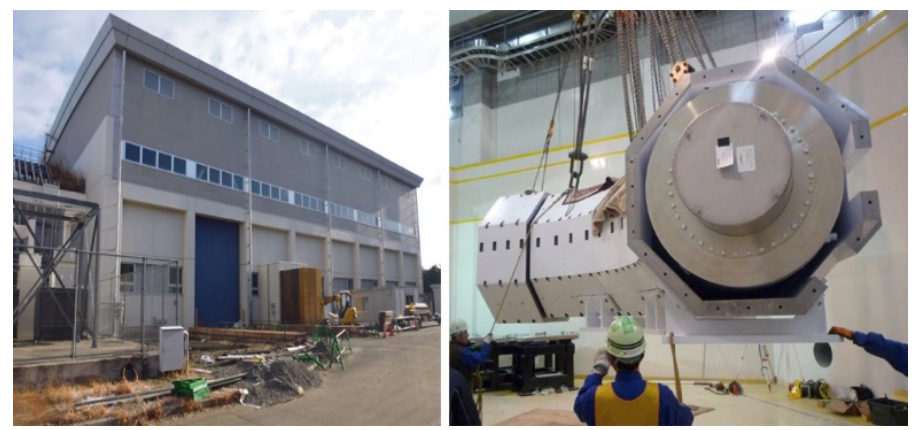

Figure 4: Photos of the present COMET Experimetal Facility; (Left) Newly constructed COMET Experimental Hall, (Right) Installation of the $90^{\circ}$ Bending Solenoid.

is extracted from the J-PARC MR and also the secondary beam line to transport the generated pion/muon from the pion production target to the COMET detector system. Figure 4 (Right) shows the installation of the $90^{\circ}$ bending solenoid for the COMET pion/muon transport line.

On the other hand, detector construction is also ongoing. The wire stringing of CDC is underway and expected to be finished by the end of 2015. The development of extremely thin wall, $20 \mu \mathrm{m}$-thick, straw-tube tracker which is operational in vacuum was successfully done, and the study of "1-to-1 size" final prototype is ongoing for assembly development and performance studies with the test beam. The mass production of this thin straw tubes, 2,500 tubes for Phase-I, were already completed. The final prototype of electromagnetic calorimeter has also been built and testbeam experiments were carried out at Tohoku university using $100 \mathrm{MeV} / c$ electron beam and at PSI using 50-100 MeV/c $\mathrm{e}^{ \pm} / \mu^{ \pm} / \pi^{ \pm}$-contaminated beam. Detector construction of straw tracker / electromagnetic calorimeter will start soon to catch up the facility construction and the Phase-I experiment.

\section{References}

[1] R. Barbieri and L.J. Hall, Phys. Lett. B338 (1994) pp.212-218

[2] T. Mori and W. Ootani, Prog. Part. Nucl. Phys. 79 (2014) pp.57-94

[3] S. Mihara, J. P. Miller, P. Paradisi and G. Pireda, Annu. Rev. Nucl. Parc. Sci. 63 (2013) pp.531-552

[4] COMET Collaboration, Conceptual Design Report for Experimental Search for Lepton Flavor Violating $\mu^{-} N \rightarrow \mathrm{e}^{-} N$ Conversion at Sensitivity of $10^{16}$ with a Slow-Extracted Bunched Proton Beam (COMET) : J-PARC P21 (KEK Report 2009-10), 2009

[5] W. H. Bertl et.al.[SINDRUM-II Collaboration], Eur. Phys. J. C47 (2006) pp.337-338

[6] R. M. Dzhilkibaev and V. M. Lobashev, Sov. J. Nucl. Phys. 49 (1989) 384

[7] N.V.Mokhov, The MARS Code System User's Guide, Fermilab-FN-628 (1995) 